\title{
The Contribution of SPECT/CT Bone Scintigraphy in the Localization of an Infective (Purulent) Sacroiliitis - A Case Report
}

\author{
Nevena Manevska**, Neron Popovski ${ }^{1}$, Tanja Makazlieva ${ }^{1}$, Hristina Popovska ${ }^{3}$, Aleksandra Pesevska-Todorcevska ${ }^{1}$, \\ Sinisa Stojanoski ${ }^{1,4}$ \\ ${ }^{1}$ Institute of Pathophysiology and Nuclear medicine, Acad. Isak S. Tadzer, Medical faculty, University of Ss Cyril and Methodius, \\ Skopje, Republic of Macedonia; ${ }^{2}$ University Clinic of Ortopedics, Medical faculty, University of Ss Cyril and Methodius, Skopje, \\ Republic of Macedonia; ${ }^{3}$ University Clinic of Neurology, Medical faculty, University of Ss Cyril and Methodius, Skopje, Republic \\ of Macedonia; ${ }^{4}$ Lazarski University, Medical faculty, Warsaw, Poland
}

Edited by: Ksenija Bogoeva-Kostovska Citation: Manevska N, Popovski N, Makazlieva Popovska H, Pesevska-Todorcevska A, Stojanoski S The Contization of an Infective (Purulent) Sacroilitis Thealization of an Infective (Purulent) Sacroilititis - A Case 2020 Sep 03; 8(C):132-139. https://doi.org/10.3889/ oamjms. 2020.4740 Keywords: Sacroilititis; SPECT/CT; bone scan; sacroiliac Correspondence: Nevena Manevska, Institute of Pathophysiology and Nuclear medicine, Acad. Isak S. Tadzer, Medical faculty, University of Ss. Cyril and Methodius, Skopje, Republic of Macedonia. Address: St. Skopje, Republic of Macedonia. E-mail: n manevskamedfukim Received: 06-Apr-2020 Revised: 09-Jun-2020 Accepted: 06-Jul-2020 Copyright: ๑ 2020 Nevena Manevska, Neron Popovski, Tanja Makazlieva, Hristina Popovska, Aleksandra Pesevska-Todorcevska, Sinisa Stojanosk

Funding: This research did not receive any financial Competing Interests: The authors have declared support competing interests exis Open Access: This is an open-access article distribute nCommercial 4.0 International License (CC BY-NC 4.0)

\begin{abstract}
BACKGROUND: Infectious sacroiliitis (ISI) is an inflammation of one or both of the sacroiliac (SI) joints, relatively rare disorder, affecting between $1 \%$ and $2 \%$ of all patients with septic arthritis. The variety of symptom presentation makes the diagnosis quite challenging. Combination of laboratory hematological tests, together with diagnostic imaging tools, such as magnetic resonance imaging (MRI), computed tomography (CT), and bone scan (BS), as well as microbiological tests contribute the final diagnosis, which may take up to several months.

CASE REPORT: We present a case of a 33-year-old male patient with a history of lower back pain with propagation of the pain in the right leg, accompanied by febrility and hematuria. Laboratory tests showed high values of C-reactive protein, high degradation products and hyperkalemia, leading to a diagnose of acute renal failure stage 3 . MRI of the lower spine and pelvis revealed hetero- signal change more to the right where the spinal canal was expanded, accumulating contrast and involved the caudate and the right radix. The displayed sequences were accompanied by an altered morphology of the spinal musculature, with intense accumulation of contrast in parts of the muscle. Paravertebral abscess was detected in the intercaudal and iliac muscles, along with inflammatory edema of the right SI with a suspicion of a sacroiliitis. One week after, a three phase BS showed positive accumulation in the right S joint in all three phases. The SI index for the right SI joint was 2.09, while for the left SI joint was 1.125. The patient underwent surgical intervention for drainage of the paravertebral abscess.

CONCLUSION: The condition of ISI may be sometimes very difficult to be recognized in many patients. Considering the diversity of the clinical manifestations, it is of great importance to select the right imaging modality. The nuclear medicine technique triple phase bone and the hybrid imaging SPECT/CT have been suggested to improve the sensitivity and specificity of the bone scan, providing better characterization of equivocal lesions, especially in the acute form for disease localization.
\end{abstract}

\section{Introduction}

Infectious sacroiliitis (ISI) is an inflammation of one or both of the sacroiliac (SI) joint, a relatively rare disorder, affecting between $1 \%$ and $2 \%$ of all patients with septic arthritis [1]. It can be caused by traumatic injury, arthritis, infection, pregnancy, or immunosuppression. The poor vascularization of the joint results in a low risk of infection through the hematogenous route. The variety of symptom presentation (lower back pain, lumbogluteal, abdominal, hip and/or posterior thigh pain, along with moving difficulty on the affected side), furthermore with the lack of symptom specificity and lack of knowledge of the diagnostic procedure, make the diagnosis quite challenging [1],[2].

Combination of laboratory hematological tests, together with diagnostic imaging tools such as magnetic resonance imaging (MRI), computed tomography (CT), and bone scan (BS), as well as microbiological tests contribute the final diagnosis, which may take up to several months. This disease is often accompanied by severe complications arising from the late diagnosis such as abscess or sequestrum formation, prolonged period of sepsis, long-term joint deformity or disability, and even death [3].

\section{Case Report}

We present a 33-year-old male patient with a history of lower back pain, with propagation of the pain in the right leg, accompanied by febrility and hematuria. The urologist thought of a bacterial uroinfection and prescribed non-steroid anti-inflammatory medication and antibiotic. Three weeks after, due to worsening of the symptoms (melena, fatigue and nausea) the patient was admitted to the hospital. Laboratory test showed high values of C-reactive protein (CRP), 
Table 1: Laboratory findings during hospitalization

\begin{tabular}{lllllll}
\hline 2018 & 29.05 & 06.06 & 09.06 & 12.06 & 18.06 & 25.06 \\
WBS & 7.5 & 5.5 & 8.3 & 5.6 & 5.4 & 5.4 \\
Lymphocytes & 0.8 & 17.5 & 10.7 & 1.1 & 23.3 & 1.2 \\
RBC & 2.85 & 3.21 & 3.2 & 3.13 & 3.32 & 3.4 \\
Hemoglobin & 86 & 89 & 90 & 91 & 95 & 97 \\
Hematocrit & 0.237 & 0.268 & 0.263 & 0.266 & 0.284 & 0.284 \\
AP & 367.22 & 191.44 & 262.08 & 187.9 & 169.06 & 156.04 \\
AST & 83.92 & 37.78 & 35.38 & 32.98 & 20.85 & 16.25 \\
ALT & 128.64 & 88.32 & 81.87 & 64.97 & 46.29 & 31.77 \\
Urea & 13.31 & 3.06 & 3.53 & 1.84 & 3.5 & 3.22 \\
Creatinine & 169.77 & 98.49 & 91.71 & 60.19 & 59.27 & 70.07 \\
Uric acid & 466.1 & 230.37 & 211.66 & 222.7 & 277.65 & 349.41 \\
CRP & 125.03 & 67.66 & 165.59 & 52.91 & 38.1 & 20.27 \\
ESR & 78 & 78 & $I$ & 80 & $l$ & 30 \\
Proteins & 72.44 & 62.28 & 57.45 & 56.81 & 59.36 & 64.88 \\
Albumin & 37.25 & 33.62 & 30.76 & 30.88 & 33.59 & 36.07 \\
Globulin & $I$ & $I$ & $I$ & 25.72 & 26.51 & $/$ \\
\hline WBS: White blood cells, RBC: Red blood cells, AP: Alkaline phosphatase, AST: Aspartate aminotransferase, \\
ALT: Alanine transaminase, CRP: C-reactive protein, ECR: Erythrocyte sedimentation rate.
\end{tabular}

high erythrocyte sedimentation rate (ESR), high degradation products, and hyperkalemia (Table 1). Due to the hematuria, renal ultrasound was performed and it showed enlarged kidneys (right kidney $132 \times$ $62 \mathrm{~mm}$, parenchyma $=25 \mathrm{~mm}$, left kidney $127 \times 63$ $\mathrm{mm}$, parenchyma $=24 \mathrm{~mm}$ ) with enlarged edema, emphasized pyramids, leading to a diagnose of an acute renal failure stage 3 . The urinoculture was positive for Pseudomonas aeruginosa: 5000/ml. Gastroscopy (for excluding upper gastrointestinal bleeding) revealed ulcer of the antrum and bulbus of the duodenum.

Clinically, the patient presented with painful limited left hip movements in all directions. He was unable to bear weight on his left leg. He had axial lower back pain and his left SI region was painful on palpation. The neurological examination did not reveal any abnormality in the inervation of the lower extremity, except the localized pain, in the left SI region, that needed further investigations. Due to the intense lower back pain, not responding to anti-inflammatory and antibiotic therapy, the patient underwent MRI of the lower spine and pelvis. It revealed hetero- signal change on the level of L4-S2, more to the right where the spinal canal is expanded. The change accumulated contrast and involved the caudate and the right radix. MRI of the pelvic region showed inflammatory edema of the Sljoint of the sacral and iliac side in term of a sacroiliitis. A larger abscess cavity was detected on the level of the right iliac muscle, with postcontrast staining, and the whole muscle was enlarged in volume. Greater abscess changes were seen on the level of $\mathrm{m}$. piriformis and $\mathrm{m}$. maximus. There was inflammatory interstitial edema to $\mathrm{m}$. gluteus minimus and medius. Furthermore, abscess formation was detected on the paravertebral level at the right sacrum level with central necrotic section with postcontrast peripheral staining. Lymph gland conglomerates were seen around the iliac blood vessels on the same side, and they were postcontrast peripherally stained, with visible necrosis. On the right femoral head, (subcapitis) a visible change was detected from the superoventral side that might had been an intraosseous abscess in the cavity (Figure 1).

The consilium review concluded paravertebral intercaudal and iliac muscle abscess and the patient was hospitalized at the Orthopedic Clinic in Skopje. Core

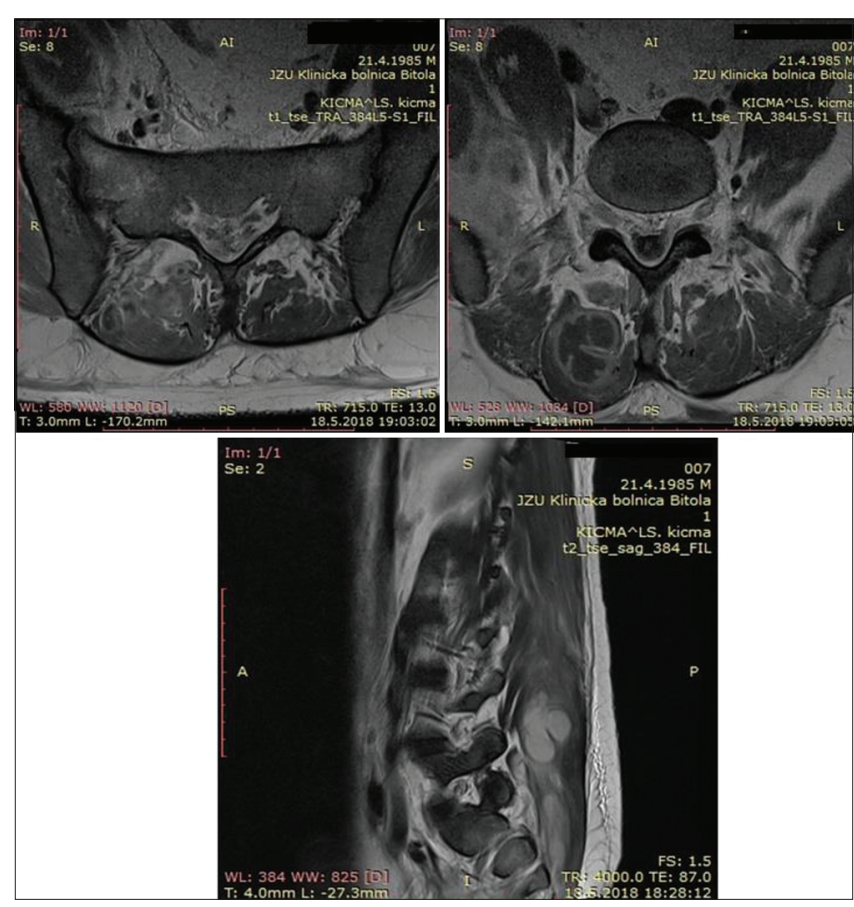

Figure 1: Magnetic resonance imaging of the lumbar spine and pelvic region

biopsy was taken from the paravertebral region and it showed regular skeletal muscle fragments, hyalinized connective tissue fragments as well as a skeletal cylinder where endomysium bundles showed proliferative connective tissue with chronic inflammatory infiltration, organized dominantly perivascular, $\mathrm{CD} 3+$ and $\mathrm{CD} 4+$.

One week after, a BS was performed that was positive in all three phases (vascular, pool phase, and late static images) in the region of the right SIJ (Figures 2 and 3 ), without any other pathologic accumulation of the osteotropic radiotracer. SI index was calculated as the ratio of average counts of whole SI regions to background region (sacral region), Figure 4 . The SI index for the right SI joint was 2.09 and for the left SIJ was 1.125 , right/left ratio $=1.861$, left/right index $=0.537$. The counts are given in Table 2. SPECT/CT (Figure 5) detected pathological accumulation in the right $\mathrm{SI}$ joint with subchondral erosions of the bone on the CT window.

\section{Table 2: Sacroiliac index (average and total counts)}

\begin{tabular}{llll}
\hline ROI counts & ROI right SIJ & ROI left SIJ & ROI sacrum \\
\hline Average counts & 26,381 & 15,355 & 13,516 \\
Total counts & $71,376,076$ & $32,137,933$ & $22,881,484$ \\
\hline
\end{tabular}

The patient underwent surgical intervention for drainage of the paravertebral abscess. The drainage was performed with left paravertebral skin incision and deep tissue dissection reaching the left SI joint. There were no skin fistulas present. The abscess pocket was recognized and drainage was made. Generous tissue irrigation was performed with saline solution and $10 \%$ iodine solution. Deep tissue drain was placed with active suction. The wound was closed by layers. The drain was not removed until full stop of the drainage. Staphylococcus aureus 


\begin{tabular}{|c|c|c|c|c|c|}
\hline \multicolumn{6}{|c|}{ Vascular phase of bone scan (AP) } \\
\hline & 1 & 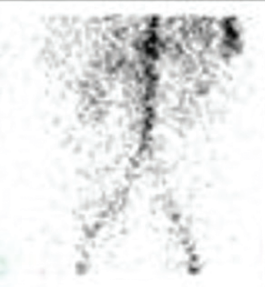 & & torstis & 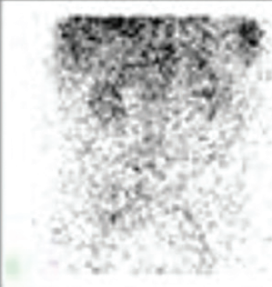 \\
\hline 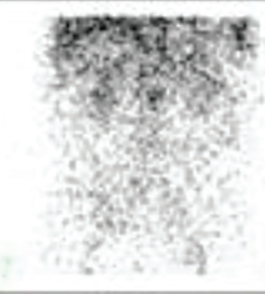 & 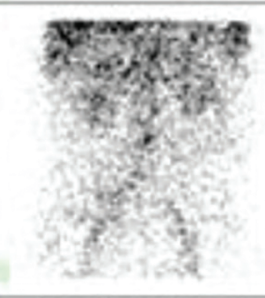 & 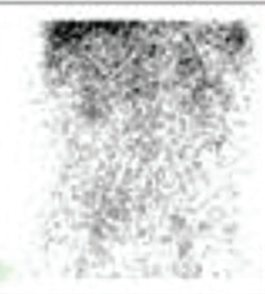 & 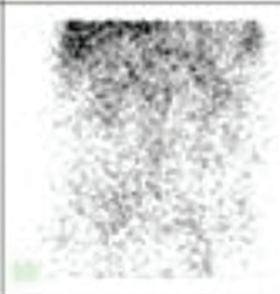 & है & 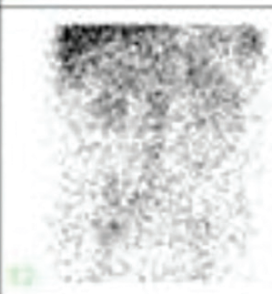 \\
\hline 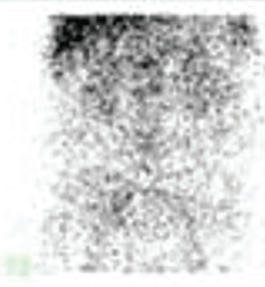 & 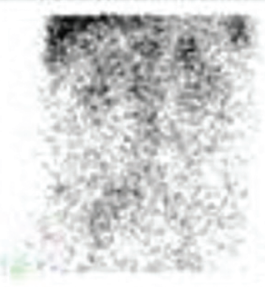 & 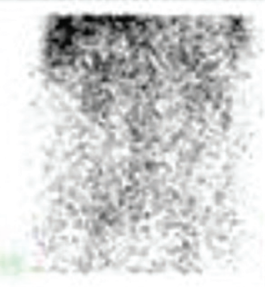 & 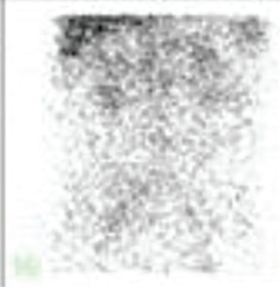 & 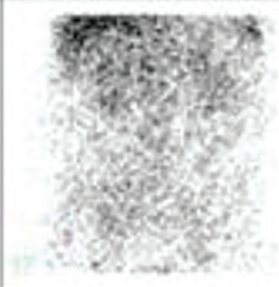 & 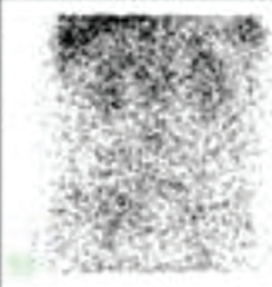 \\
\hline 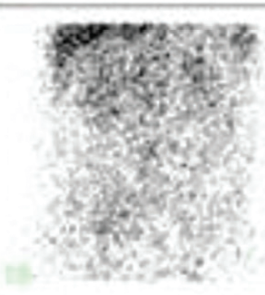 & 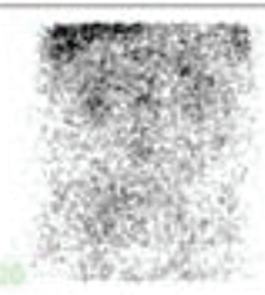 & 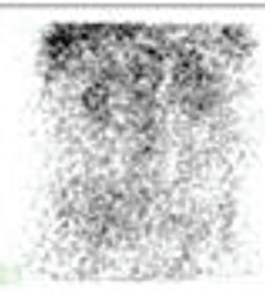 & 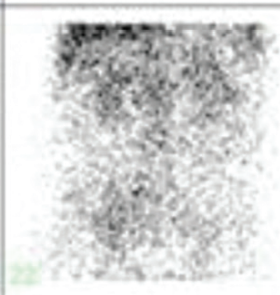 & 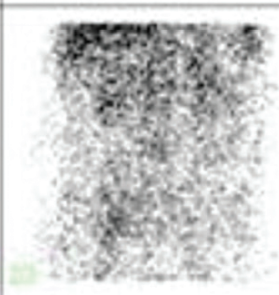 & 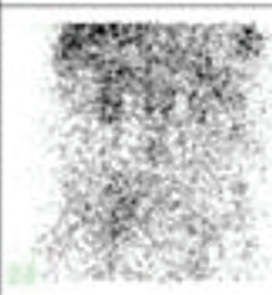 \\
\hline 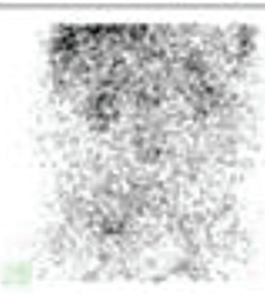 & 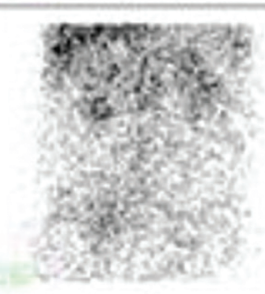 & 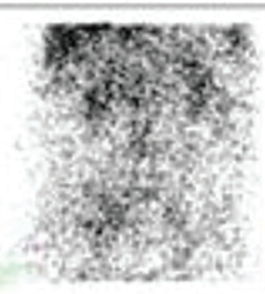 & 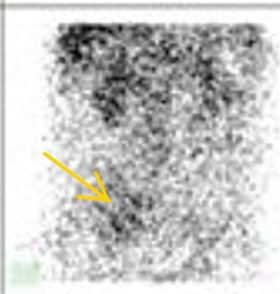 & 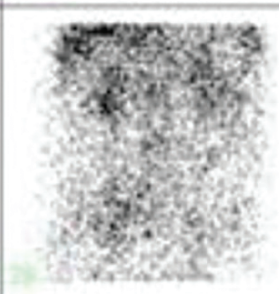 & 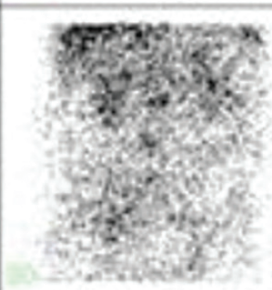 \\
\hline
\end{tabular}

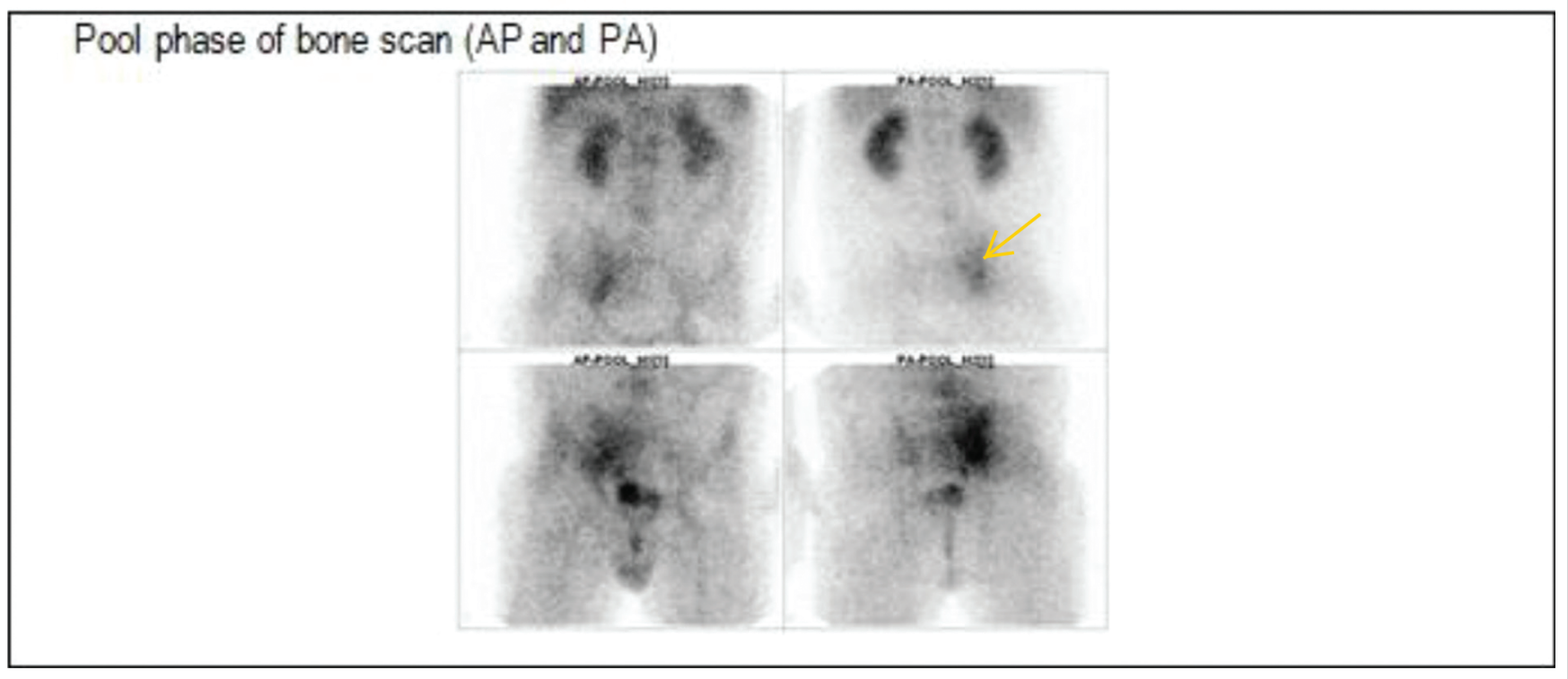

Figure 2: Bone scan (vascular and pool phase), right SI joint (yellow arrow) 


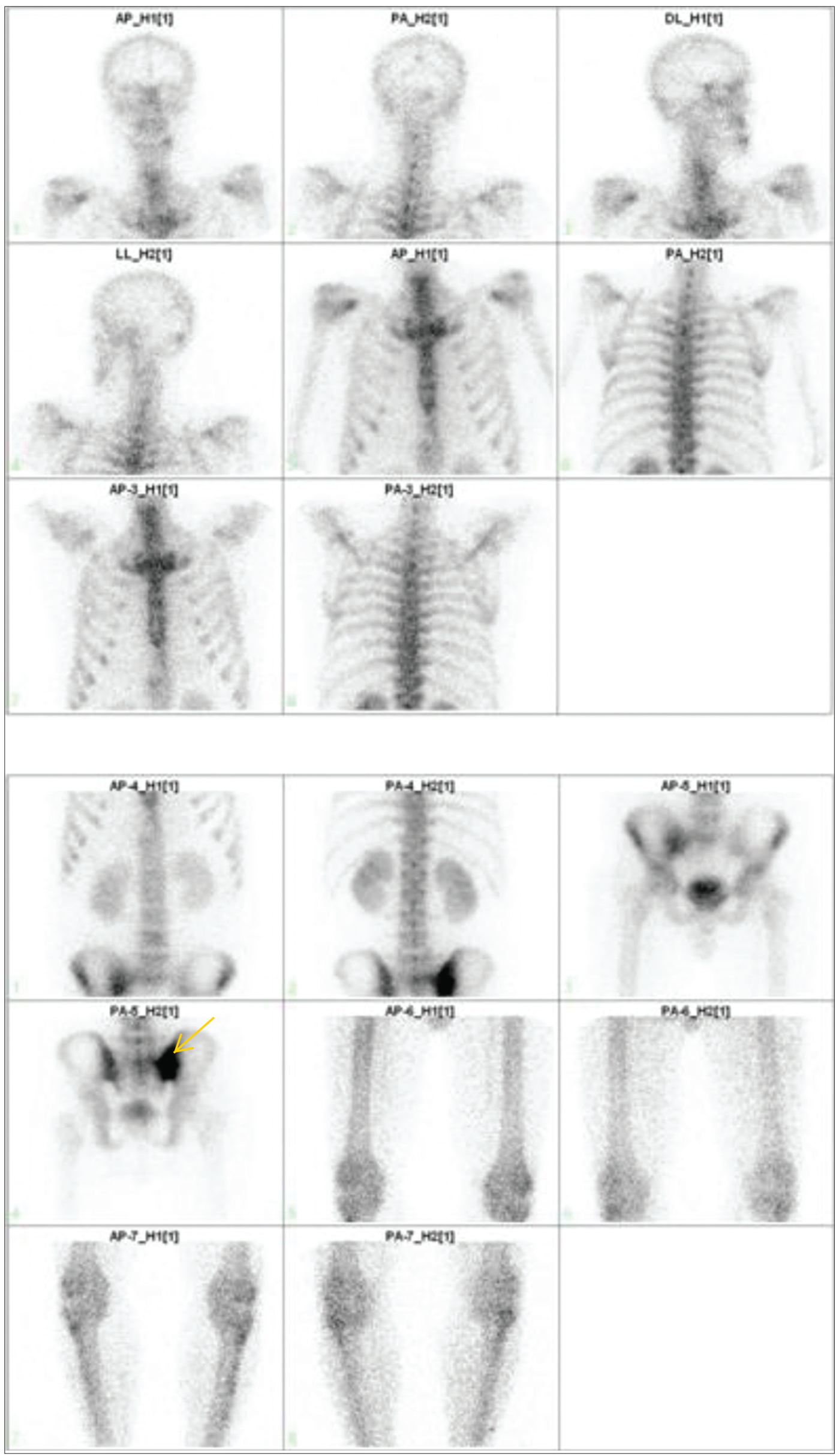

Figure 3: Bone scan (late static images), right SI joint (yellow arrow) 


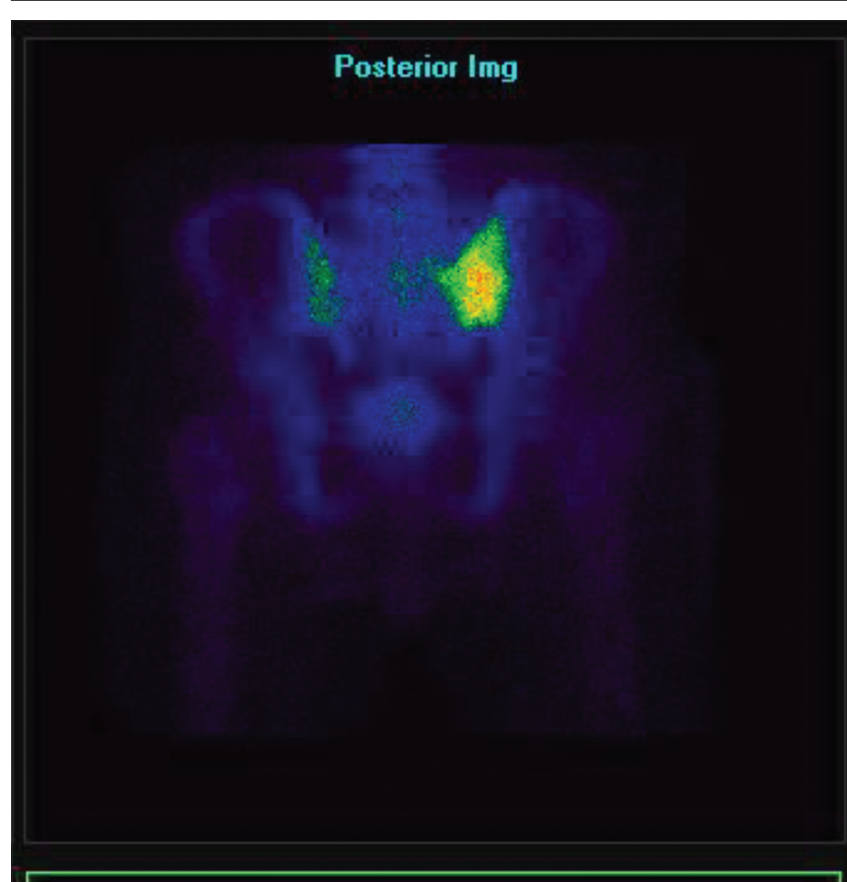

Posterior Img Filtered None

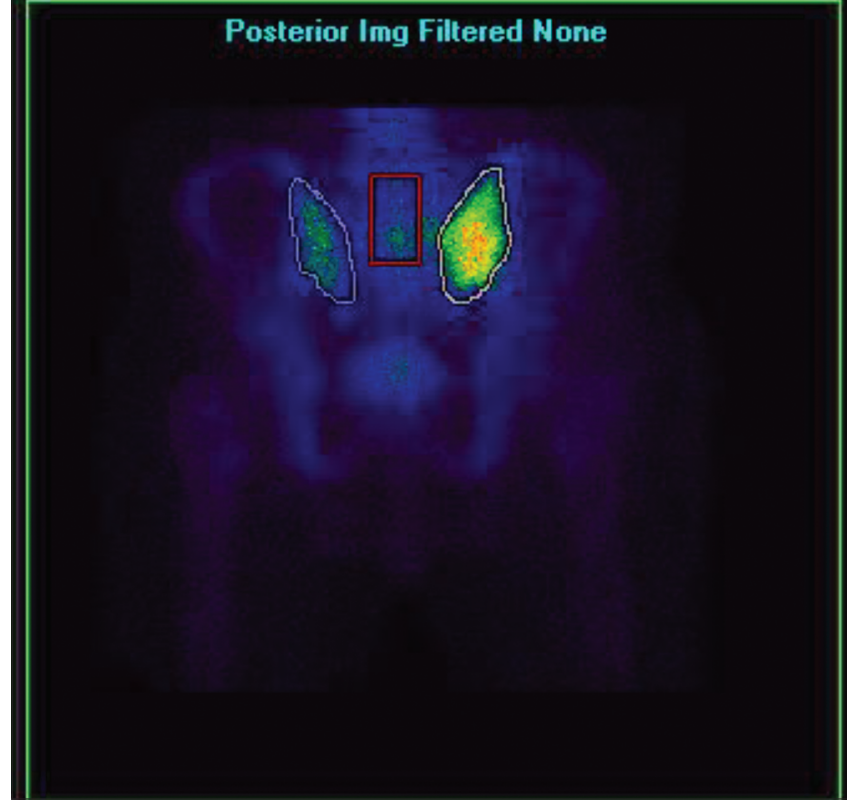

Figure 4: Sacroiliac index region of interests

was isolated. He had i.v antibiotics for 10 days in the post-operative period during hospitalization. He was discharged from the hospital with less pain and advised to continue the antibiotic treatment in the next 6 weeks per os. He was still unable to bear weight, so he was advised to use crutches for the next month. Gradually, clinically and laboratory his condition was getting better, with lowering of the CRP. On the follow-up, three months after, he was pain free and walking without crutches.

\section{Discussion}

ISI is considered to be an uncommon condition. It can be seen in patients of any age mainly in elderly, with higher incidence in female than male patients [2]. Infectious arthritis of any joint has a prevalence of 2-10 per 100,000 people. In $50 \%$ of cases, the knee is the joint most commonly affected, followed by the hip, shoulder, and elbow [4]

A good anamnesis and some clinical factors may guide the bacteriological investigation. Polynuclear neutrophil leukocytosis in addition to increased levels of CRP and ESR are standard features of ISI, while being inconsistent and non-specific. The definitive microbiological diagnosis may be based on blood cultures, joint fluid by CT-guided percutaneous puncture or surgical investigations.

The underlying pathophysiology of ISI is thought to involve hematogenous spread of bacteria or local extension of adjacent soft tissue or bone infection. The most common isolated culprit is $S$. aureus; however, other bacteria can be identified as well such as Salmonella, Streptococcus, Brucella, Mycobacterium tuberculosis and Pseudomonas [5].

Our case showed young patient in the acute stage of the disease, immunocompromised patient, with acute renal insufficiency that showed isolated $S$. aureus from the surgical procedure.

In the study of Wu et al. in a 10-year period (1996-2005), 33 patients were diagnosed with pyogenic sacroiliitis. They had S. aureus, accounting for $80 \%$ of blood culture isolates in pediatric patients and $50 \%$ in adults. Group B Streptococcus and Salmonella species were cultured as well [2].

The retrospective study of Hermet included 39 ISI cases, diagnosed in French rheumatology departments. Sacroiliitis was confirmed bacteriologically or, in the absence of pathogenic agents, if clinical, biological, and radiological data were compatible with this diagnosis, having favorable evolution under antibiotic therapy [1].

For differentiating the nature of the sacroiliitis - infective from non-infective (inflammatory), three features may be present. Intense bone marrow edema may appear as a first sign in ISI with more intraarticular fluid. Afterward, involvement of the periarticular soft tissues with inflammation, particularly the iliac and gluteal muscles and periarticular fluid collection or abscess is the pathognomonic sign of an infective sacroiliitis [6]

Prominent SI joint uptake and the increased SI ratio in BS generally portray an advanced stage of sacroiliitis. However, the SI ratio measured in bone scintigraphy uses a rectangular region of interest that does not represent the accurate uptake of the SI joint and sacrum. Bone scintigraphy as a screening tool in diagnosis of sacroiliitis was performed in the '90s, but the literature showed very low sensitivity of $52 \%$ in patients without radiographic changes and $66 \%$ in patients with ankylosing spondylitis and Grade 2 or 3 sacroiliitis [7] 

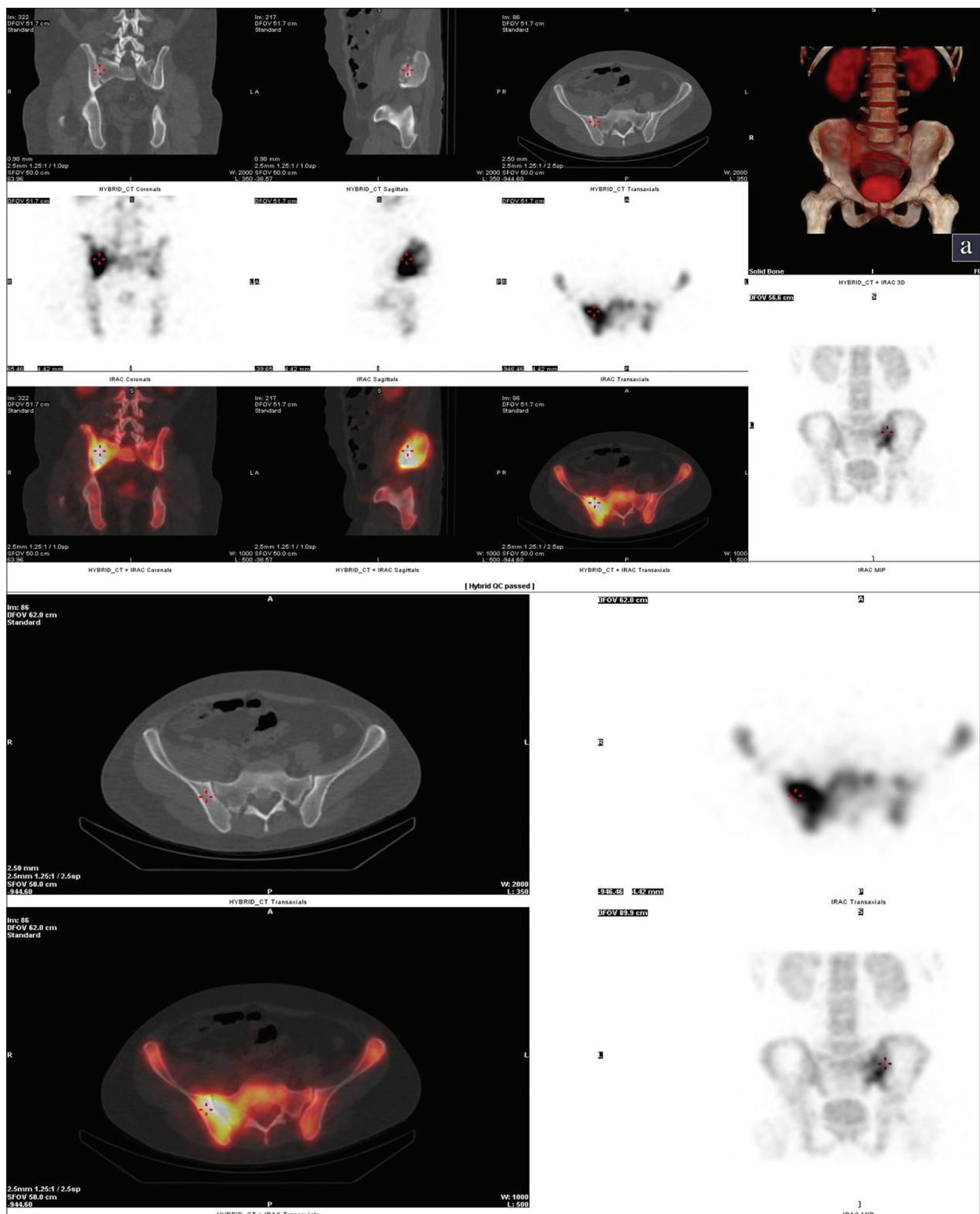

Technetium (Tc-99m) BS is more sensitive for the diagnosis of ISI than plain radiography and may be positive as early as three days after the onset of symptoms. Gallium-67 citrate scanning was shown to demonstrate uptake positivity earlier than Tc- $99 \mathrm{~m} \mathrm{BC}$ in a group of drug users with septic arthritis [8]. However, taking into consideration of the low rates of sensitivity of the planar bone scintigraphy clinicians have limited the use of scintigraphy as a diagnostic differential tool in sacroiliitis patients. Since it is of great importance to differentiate the two entities of ISI and spondyloarthritis, while the therapeutic approach is different, MRI was accepted as a gold standard method for detection of ISI as well as follow-up of the patients (of their clinical results and/or additional result of the morphologic imaging method). When MRI gives low signal intensity on T1-weighted images and high signal intensity on T2-weighted images, then ISI is suspected. In our patient, MRI showed mild expansion of the infected SI joint space along with effusion and bone marrow edema on the joint surfaces, indicating active inflammation. Some features characterizing the sacroiliitis were described by Sturzenbecher and coauthors, such as subperiosteal infiltration, transcapsular infiltrations of juxtaarticular muscle layers on MRI [9].

In the chronic phase, MRI can show periarticular bone marrow reconversion, replacement of articular cartilage by pannus, bone erosion, subchondral sclerosis, joint space widening or narrowing, and ankylosis [10]. Our patient displayed altered morphology of the spinal musculature on the MRI with intense accumulation of contrast in parts of the muscle. CT however, can be helpful in visualizing associated abscesses and guiding arthrocentesis. The most frequent findings of sacroiliitis on CT are: joint space narrowing, subchondral sclerosis, bone erosions, and ankylosis. 
In the case of our patient, MRI study showing big abscess and inflammatory edema of the SI joint, was in favor of sacroiliitis, but additionally bone SPECT/CT images was made, that confirmed the acute phase of the disease and also localized the infection of the SI joint on the right side, excluding other pathological accumulation (other sites of infection). Higher sensitivity of $85 \%$, but lower specificity of $47 \%$ and positive predictive value of $60 \%$ of MRI for sacroiliitis was presented by Oostveen et al. [11]. One retrospective analysis of 9 patients, average age 27.3 years, diagnosed with acute pyogenic infection of the SI joint, showed positive MRI in all cases, while BS was positive in 6 cases [12]. Klein et al. stated that in six septic SI joints studied, all had positive MRI, five had positive gallium scans, three had positive CT scans, and one had a positive Tc-99m BS [13].

During the years, the new techniques of SPECT and furthermore the hybrid imaging of SPECT/ CT increased the sensitivity of bone scintigraphy and the role of this imaging modality in sacroiliitis patients.

Cusi et al. used the hybrid imaging with SPECT/ $\mathrm{CT}$, investigated patients with peripartum pain lasting more than 2 years, and found metabolic alterations around the SI joint in patients with SI joint dysfunction and revealed $95 \%$ sensitivity and $99 \%$ specificity [14].

The role of imaging studies in the evaluation of patients with sacroiliitis is controversial. Sensitivity values were $25 \%, 47 \%, 69 \%$ and $97 \%$ and specificity values were $80 \%, 90 \%, 95 \%$ and $90 \%$ in quantitative bone scanning, $99 \mathrm{mTc}-\mathrm{BS}$, planar and SPECT bone imaging, respectively, when the clinical findings were considered as the "gold standard" [15].

Low specificity of bone scintigraphy in diagnosis of sacroiliitis, despite performing SPECT imaging was presented in the study of Koc. However, calculation of index values increased the specificity of scintigraphy, $73 \%$ versus $97 \%$ [16]. Our study also confirmed pathologic value of the SI index, on the side of the sacroiliitis.

In the study of Song et al., SPECT/CT compared with conventional BS was found to be more useful method to detect SI changes in early phase to differentiate the subtle changes of sacroiliitis by plain radiography in contrast to $B S$ [7].

Additional benefit of the BS was presented in the study of Gheita, where BS was able to explain unrecognized articular and entheseal involvement in patients with septic arthritis [17].

The main disadvantages in performing a BS are: The inability to differentiate infectious from nonISI, the inability to differentiate sacroiliitis from psoas or gluteal abscess, and the inability to identify spread of the infection from the joint into the surrounding tissues. Though, with the BS we performed in our patient we showed ISI that is localized in one location - the sacroiliac joint.
Recently, positron emission tomography/CT with fluorine-18 fluorodeoxyglucose appeared to be an interesting technique for the very early diagnosis of ISI, even before MRI reveals any abnormalities [1].

On the other hand, Battafarano et al. found higher sensitivity and specificity $(100 \%)$ for detecting sacroiliitis with MRI Type I alterations, and, respectively $69 \%$ and $46 \%$, in the evaluation of Type II alterations [18] . The main disadvantages of MRI are: high cost, low availability, and long duration of examination (30 min), although the latter is well tolerated by the majority of patients, in pediatric examinations included in the study.

Scott presented similar case of a young 21-year-old boy who was admitted at the emergency department due to left hip pain and inability to walk. MRI in T2 presented enhancing fluid in the left SI joint communicating with enhancing fluid anterior to the $\mathrm{SI}$ joint. Irregularity of the edges of SI joint was also noted [19].

So far, no consensus exists for duration of antibiotic treatment for ISI. The recommended duration of treatment is at least a 2-week course of IV antibiotics followed by a 6 -week course of oral antibiotics [1].

\section{Conclusion}

The condition of ISI may be sometimes very difficult to be recognized in many patients. Considering the diversity of the clinical manifestations, it is of great importance to select the right imaging modality. The MRI plays an essential role in better demonstrating early alterations and inflammatory activity of the process. On the other hand, CT is superior in quantifying chronic alterations. The nuclear medicine technique three phase bone SPECT/CT has been suggested to improve the sensitivity and specificity of the BS, providing better characterization of equivocal lesions, especially in the acute form for disease localization.

\section{References}

1. Hermet M, Minichiello E, Flipo RM, Dubost JJ, Allanore Y, Ziza JM, et al. Infectious sacroiliitis: A retrospective, multicentre study of 39 adults. BMC Infect Dis. 2012;12:305. https://doi. org/10.1186/1471-2334-12-305

PMid:23153120

2. Wu MS, Chang SS, Lee SH, Lee CC. Pyogenic sacroiliitis-a comparison between pediatric and adult patients. Rheumatology. 2017;46(11):1684-7. https://doi.org/10.1093/rheumatology/ kem201

PMid:17901064

3. Cinar M, Sanal HT, Yilmaz S, Simsek I, Erdem H, Pay S, et al 
Radiological follow up of the evolution of inflammatory process in sacroiliac joint with magnetic resonance imaging: A case with pyogenic sacroiliitis. Case Rep Rheumatol. 2012;2012:509136. https://doi.org/10.1155/2012/509136

PMid:23050188

4. Abelson A. Septic Arthritis. Cleveland Clinic; 2010. Available from: http://www.clevelandclinicmeded.com/medicalpubs/ diseasemanagement/rheumatology/septic-arthritis. [Last accessed on 2016 Oct 28].

5. Malhotra $A$, Kalil $R$, Jones $R$, Schwartz $D$, Qadeer $A H$, Huang $M$, et al. Infectious sacroiliitis: A radiographic no touch lesion. J Gen Pract (Los Angel). 2017;5:336. https://doi. org/10.4172/2329-9126.1000336

6. Tsoi C, Griffith JF, Lee RK, Wong PC, Tam LS. Imaging of sacroiliitis: Current status, limitations and pitfalls. Quant Imaging Med Surg. 2019;9(2):318-335https://doi.org/10.21037/ qims.2018.11.10

PMid:30976556

7. Song IH, Carrasco-Fernandez J, Rudwaleit M, Sieper J. The diagnostic value of scintigraphy in assessing sacroilitis in ankylosing spondylitis: A systematic literature research. Ann Rheum Dis. 2008;67(11):1535-40. https://doi.org/10.1136/ ard.2007.083089

PMid:18230629

8. Morsi A, Sallam A, Saoud A. Infectious sacroiliitis (ISI). World Spinal Column J. 2015;6(1): 27-35

9. Stürzenbecher A, Braun J, Paris S, Biedermann T, Hamm B, Bollow M. MR imaging of septic sacroiliitis. Skeletal Radiol. 2000;29(8):439-46. https://doi.org/10.1007/s002560000242

PMid:11026711

10. Ahmed H, Siam AE, Gouda-Mohamed GM, Boehm H. Surgical treatment of sacroiliac joint infection. J Orthopaed Traumatol 2013;14(2):121-9. https://doi.org/10.1007/s10195-013-0233-3 PMid:23558792

11. Oostveen J, Prevo R, den Boer J, van de Laar M. Early detection of sacroiliitis on magnetic resonance imaging and subsequent development of sacroilitis on plain radiography. J Rheumatol. 1999;26(9):1953-8.

\section{PMid: 10493676}

12. Doita $M$, Yoshiya $S$, Nabeshima $Y$, Tanase $Y$, Nishida $K$, Miyamoto $\mathrm{H}$, et al. Acute pyogenic sacroiliitis without predisposing conditions. Spine (Phila Pa 1976). 2003;28(18):E384-9. https:// doi.org/10.1097/01.brs.0000092481.42709.6f

PMid: 14501940

13. Klein MA, Winalski CS, WaxMR, Piwnica-Worms DR. MR imaging of septic sacroiliitis. J Comput Assist Tomogr. 1991;15(1):12632. https://doi.org/10.1097/00004728-199101000-00020 PMid:1987181

14. Cusi M, Saunders J, Van der Wall H, Fogelman I. Metabolic disturbances identified by SPECT-CT in patients with a clinical diagnosis of sacroiliac joint incompetence. Eur Spine J. 2013;22(7):1674-82. https://doi.org/10.1016/j.joca.2012.02.368 PMid:23455953

15. Yildiz A, Gungor F, Tuncer T, Karayalcin B. The evaluation of sacroilitis using 99mTc-nanocolloid and 99mTc-MDP scintigraphy. Nucl Med Commun. 2001;22(7):785-94. https:// doi.org/10.1097/00006231-200107000-00010

PMid: 11453052

16. Koç ZP, CengizAK, Aydın F, Samancı N, Yazısız V, Koca SS, et al Sacroiliac indicis increase the specificity of bone scintigraphy in the diagnosis of sacroiliitis. Mol Imaging Radionucl Ther. 2015;24(1):8-14. https://doi.org/10.4274/mirt.40427 PMid:25800592

17. Gheita TA, Azkalany GS, Kenawy SA, Kandeel AA. Bone scintigraphy in axial seronegative spondyloarthritis patients: Role in detection of subclinical peripheral arthritis and disease activity. Int J Rheum Dis. 2015;18:553-9. https://doi. org/10.1111/1756-185x.12527

18. Battafarano DF, West SG, Rak KM, Fortenbery EJ, Chantelois AE. Comparison of bone scan, computed tomography, and magnetic resonance imaging in the diagnosis of active sacroiliitis. Semin Arthrit Rheum. 1993;23(3):161-76. https://doi.org/10.1016/ s0049-0172(05)80037-x

PMid:8122119

19. Scott KR, Rising KL, Conlon LW. Infectious sacroiliits. J Emerg Med. 2014;47(3):83-4. 